\title{
Optimal power allocation for complex field network coding scheme with the $K$-th best relay selection
}

\author{
Xi CAI*, Pingzhi FAN, Qingchun CHEN \\ Institute of Mobile Communications, Southwest Jiaotong University, Chengdu 610031, China
}

\begin{abstract}
Wireless relay and network coding are two critical techniques to increase the reliability and throughput of wireless cooperative communication systems. In this paper, a complex field network coding (CFNC) scheme with the $K$-th best relay selection (KBS) is proposed and investigated, wherein the $K$-th best relay is selected to forward the multiplexed signal to the destination. First, the upper bound of the symbol error probability (SEP), the diversity order, and the coding gain are derived for the CFNC scheme with KBS. Then, the coding gain is utilized as the optimized criterion to determine the optimal power allocation. It is validated through analysis and simulation that the CFNC scheme with KBS can achieve full diversity only when $K=1$, while the diversity order decreases with increasing parameter $K$, and the optimal power allocation can significantly improve the performance of the CFNC scheme with KBS.
\end{abstract}

Key words: complex field network coding; the $K$-th best relay selection; power allocation

(C) 2012 JMT. All rights reserved.

\section{Introduction}

$\mathrm{C}$ ooperative transmissions have been extensively utilized to provide spatial diversity and enhance the performance of wireless networks [1-2]. The multiple-access multiple-relay (MAMR) model represents a typical cooperative communication scenario wherein multiple sources deliver their messages to one common destination with the assistance of multiple relays. The introduction of network coding can be employed to further improve the spectral efficiency by allowing relay(s) to multiplex the received data from multiple sources [3]. In the MAMR with network coding (MAMR-NC) system [3-4], communication can be carried out in two phases, namely, the multiple access (MA) phase and the relaying phase. In the MA phase, all sources will transmit their data to the relay nodes. In the relaying phase, the relays will forward the received signal from multiple sources to the destination.

Basically, there are two categories of network coding according to the transmission modes in the MA phase. The first one is algebraic network coding over a finite field [5-9], in which every source transmits its data to the relay(s) via orthogonal channels in the MA phase. The second one is the real- or complex- valued network

Received Oct. 21, 2012; revision accepted Nov. 15, 2012

${ }^{*}$ Corresponding author. Tel.:+86-13880484667

E-mail: caixi-1983@163.com (X. CAI)

(C) 2012 JMT. All rights reserved

doi: 10.3969/j.issn.2095-087X.2012.04.010 coding, which includes physical layer network coding (PNC) [10], analog network coding (ANC) [11], and complex field network coding (CFNC) [3-4]. Compared with the algebraic network coding, PNC, ANC, and CFNC allow multiple sources to transmit in a nonorthogonal manner to the relay(s) so as to improve spectral efficiency in the MA phase while keeping the full cooperative diversity. To overcome this limitation, multiuser detection [12-14] was proposed to enable multiple sources to share a channel during the MA phase. By incorporating CFNC with multiuser detection [3-4], the spectral efficiency can be greatly improved for the multiple source cooperative network.

For the multiple-relay cooperative communication system, it has been shown that efficient relay selection (RS) in cooperative networks can be utilized to improve the system performance [15-19]. In one design [3], the best relay node which provides the best SNR at the destination is selected to forward the source signals to the destination. However, in practical applications, the best relay may be unavailable due to different reasons [1819]. For example, the selected relay is running out of transmit power, or the best relay is not available due to scheduling constraints. In this case, the second best relay, or more generally the $K$-th best relay, can instead be selected to forward the network-coded signals to the destination.

In this paper, we investigate the CFNC scheme with the $K$-th best relay selection (KBS). First, the symbol error probability (SEP) of the CFNC scheme with KBS is 
presented. Then, the optimal power allocation in terms of the minimized SEP is derived. Finally, simulations are conducted to corroborate that the CFNC scheme with KBS can be improved by using the optimal power allocation and the achieved diversity order is $(M-K+1)$, where $M$ is the number of the relays.

The following notations are used throughout the paper: the complex conjugate, hermitian and determinant of a matrix are denoted by $(\cdot)^{*},(\cdot)^{\mathrm{H}}$ and $\operatorname{det}(\cdot)$, respectively; $\operatorname{diag}(\cdot)$ denotes a diagonal matrix; $\boldsymbol{I}$ is the identity matrix; $E[\cdot]$ denotes the statistical expectation; $|\mathcal{A}|$ denotes the cardinality of set $\mathcal{A} ;\|\boldsymbol{x}\|$ is the $l_{2}$ norm of $\boldsymbol{x} ;($.$) !! is$ double factorial; $\mathcal{C N}\left(0, \sigma^{2}\right)$ represents the circularlysymmetric Gaussian random distribution with mean $u$ and variance $\sigma^{2}$; and $\mathbb{S}$ denotes the universal set.

\section{System model}

Let us consider a general multiple relay cooperative transmission model with $N$ sources $\left\{S_{n}, n \in\{1,2, \cdots, N\}\right\}, M$ relays $\left\{R_{m}, m \in\{1,2, \cdots, M\}\right\}$, and one common destination $D$, which is represented by the triplet $(N, M, 1)$ [3]. It is assumed that perfect CSI is available at all relays and the destination.

In the MA phase, all $N$ source nodes transmit simultaneously to the relays and destination, where perfect synchronization among all transmitters is assumed. $\beta_{S} \theta_{S_{n}} x_{n}$ represents the transmitted signal by $S_{n}$, where $x_{n}$ is the modulated symbol, $\theta_{S_{n}}$ represents the CFNC coding coefficient, while $\beta_{S}$ is a transmit power scaling factor at the sources. The modulated symbol $x_{n} \in C_{n}$ and $E\left[\left\|x_{n}\right\|^{2}\right]=1$, where $C_{n}$ is the constellation set at $S_{n}$. Moreover, $x_{n}, \theta_{S_{n}}$, and $\beta_{S}$ should satisfy the transmit power constraint at $S_{n}$, i.e., $E\left[\left\|\beta_{S} \boldsymbol{l}_{N} \boldsymbol{\theta}_{S} \boldsymbol{x}\right\|^{2}\right]=1$, where $\boldsymbol{\theta}_{S}=\operatorname{diag}\left(\theta_{S_{1}}, \theta_{S_{2}}, \cdots, \theta_{S_{N}}\right), \boldsymbol{x}=\left[x_{1}, x_{2}, \cdots, x_{N}\right]^{\mathrm{T}}$. So the received signal at node $Q\left(Q \in\left\{R_{m}, D\right\}\right)$ in the MA phase is

$$
y_{Q}^{(1)}=\sqrt{\alpha P} \beta_{S} \boldsymbol{h}_{S Q} \boldsymbol{\theta}_{S} \boldsymbol{x}+n_{Q}^{(1)},
$$

where $P$ denotes the total transmit power in both the MA and the relaying phases. The transmit power in the MA phase and the relaying phase are $\alpha P$ and $(1-\alpha) P$, respectively $(0<\alpha<1) . \boldsymbol{h}_{S Q}=\left[h_{S_{1} Q}, h_{S_{2} Q}, \cdots, h_{S_{N} Q}\right]$ denotes the channel coefficient vector from the $N$ sources to node $Q$. Without loss of generality, $h_{S_{n} Q}(1 \leq n \leq N)$ represent i.i.d. random variables (RVs) with $h_{S_{n} Q} \sim \mathcal{C N}\left(0, \sigma_{S Q}^{2}\right) . n_{Q}^{(1)} \sim \mathcal{C N}\left(0, N_{0}\right)$ is the noise.

After the MA phase, $R_{m}$ would detect the received signals from the $N$ sources according to the following maximum likelihood (ML) decoding method:

$$
\hat{\boldsymbol{x}}_{R_{m}}=\underset{\boldsymbol{x} \in \mathcal{A}_{x}}{\arg \min }\left\|y_{R_{m}}^{(1)}-\sqrt{\alpha P} \beta_{S} \boldsymbol{h}_{S R_{m}} \boldsymbol{\theta}_{S} \boldsymbol{x}\right\|^{2},
$$

where $\mathcal{A}_{x} \triangleq\left\{\left[x_{1}, x_{2}, \cdots, x_{N}\right]: x_{n} \in C_{n}\right\}$, the number of set $\left|\mathcal{A}_{x}\right|$ is

$$
\left|\mathcal{A}_{x}\right|=\prod_{n=1}^{N}\left|C_{n}\right| .
$$

In [3-4], the detection mechanism at the destination requires the CSI of the whole network, which may cause heavy signaling overhead. In order to reduce the overhead and to counteract the error propagation problem, it is assumed that only the relays which can fully decode all the source messages will forward the received data to the destination in this paper. In the relaying phase, the $K$-th best relay in the decoding set $\mathcal{M}$ $\left(\mathcal{M}=\left\{m \mid \hat{\boldsymbol{x}}_{R_{m}}=\boldsymbol{x}, 0 \leq m \leq M\right\}\right)$, which is defined as the set of relays that can correctly decode all the source messages, performs network coding over a complex field, and forwards the network-coded signal to $D$. We define that the $K$-th best relay is selected according to the following criterion:

$$
\left\|h_{R_{w} D}\right\|^{2}=\arg K^{\text {th }} \max _{m \in \mathcal{M}}\left\|h_{R_{m} D}\right\|^{2} .
$$

Specifically, the transmitted messages in the relaying phase will be $\beta_{R} \boldsymbol{\theta}_{R} \boldsymbol{x}$, where $\boldsymbol{\theta}_{R}=\left[\theta_{R_{1}}, \theta_{R_{2}}, \cdots, \theta_{R_{N}}\right]$ denotes the CFNC coding coefficient vector at each relay, $\beta_{R}$ should satisfy the transmit power constraint at the relays, i.e., $\left.E\left[\left\|\beta_{R} \boldsymbol{\theta}_{\boldsymbol{R}} \boldsymbol{x}\right\|^{2}\right]=1 . h_{R_{m} Q}(1 \leq m \leq|\mathcal{M}|)\right)$ ) are assumed to be i.i.d. RVs. $h_{R_{m} D} \sim \mathcal{C N}\left(0, \sigma_{R D}^{2}\right)$ represents the channel coefficient between $R_{m}$ and $D$. The received signal at $D$ is

$$
y_{D}^{(2)}=\sqrt{(1-\alpha) P} h_{R_{w} D} \beta_{R} \boldsymbol{\theta}_{R} \boldsymbol{x}+n_{D}^{(2)},
$$

where $n_{D}^{(2)} \sim \mathcal{C N}\left(0, N_{0}\right)$ denotes the noise.

The destination will try to restore the message from the received signals in both phases by using the following ML decoding method

$$
\begin{gathered}
\hat{\boldsymbol{x}}_{D}=\underset{\boldsymbol{x} \in \mathcal{A}_{x}}{\arg \min }\left\{\left\|y_{D}^{(1)}-\sqrt{\alpha P} \beta_{S} \boldsymbol{h}_{S D} \boldsymbol{\theta}_{S} \boldsymbol{x}\right\|^{2}+\right. \\
\left.\left\|y_{D}^{(2)}-\sqrt{(1-\alpha) P} \beta_{R} h_{R_{w} D} \boldsymbol{\theta}_{R} \boldsymbol{x}\right\|^{2}\right\} .
\end{gathered}
$$




\section{Performance evaluation of the CFNC scheme with KBS}

Without loss of generality, each $\boldsymbol{x} \in \mathcal{A}_{x}$ is assumed to be transmitted with the same probability, where $\boldsymbol{x}$ is seen as one symbol. Let that

$$
\begin{aligned}
& t_{1}=\sum_{n=1}^{N}\left\|\theta_{S_{n}}\left(x_{n}-\tilde{x}_{n}\right)\right\|^{2}, \quad t_{2}=\left\|\boldsymbol{\theta}_{R}(\boldsymbol{x}-\tilde{\boldsymbol{x}})\right\|^{2}, \\
& t_{R}=\sum_{\forall \hat{\boldsymbol{x}}_{R_{m}} \in \mathcal{\mathcal { A }}_{x}, \hat{\boldsymbol{x}}_{R_{m}} \neq \boldsymbol{x}}\left(\sum_{n=1}^{N}\left\|\theta_{S_{n}}\left(x_{n}-\hat{x}_{R_{m}, n}\right)\right\|^{2}\right)^{-1},
\end{aligned}
$$

and

$$
f(i)=\frac{\Gamma(i-K+1) 4^{i-K+2} i ![2(i-K)+3] ! !}{2 t_{1} \alpha \beta_{S}^{2} \sigma_{S D}^{2}\left[t_{2}(1-\alpha) \beta_{R}^{2} \sigma_{R D}^{2}\right]^{i-K+1}(i-K) !(K-1) ![2(i-K+2)] ! !} .
$$

We have the following theorem.

Theorem 1 The SEP at $D$ is bounded by

$$
P_{e} \leq \min \left\{1,\left(G_{c} \cdot \bar{\gamma}\right)^{-(M-K+2)}\right\} .
$$

For the CFNC scheme with KBS, the diversity order is $M-K+2$, while the coding gain is given by

$$
G_{c}=\left(\frac{1}{\left|\mathcal{A}_{x}\right|} \sum_{\forall \boldsymbol{x}, \tilde{\boldsymbol{x}} \in \mathcal{A}_{x}, \boldsymbol{x} \neq \tilde{\boldsymbol{x}}} \kappa_{1, \boldsymbol{x} \rightarrow \tilde{\boldsymbol{x}}}\right)^{-\frac{1}{M-K+2}}
$$

where

$$
\begin{aligned}
& \kappa_{1, x \rightarrow \tilde{x}} \approx \frac{\bar{\gamma} \gg 1}{t_{1}\left(\alpha \beta_{S}^{2}\right)^{M-K+2} \sigma_{S D}^{2} \sigma_{S R}^{2(M-K+1)}}+ \\
& \sum_{i=K}^{M} \frac{\left(\begin{array}{l}
M \\
K-1
\end{array}\right) t_{R}^{M-i} f(i)}{\left(\alpha \beta_{S}^{2} \sigma_{S R}^{2}\right)^{(M-i)}} .
\end{aligned}
$$

\section{Proof:}

The SEP at $D$ is upper bounded by

$$
\begin{aligned}
P_{\mathrm{e}} \leq & \frac{1}{\left|\mathcal{A}_{x}\right|} \times \\
& \sum_{\substack{\forall \boldsymbol{x}, \tilde{\boldsymbol{x}} \in \mathcal{A}_{x} \\
\boldsymbol{x} \neq \tilde{\boldsymbol{x}}}} E_{\boldsymbol{h}_{S D}, \boldsymbol{H}_{S R}, \boldsymbol{h}_{R D}}\left[\operatorname{Pr}\left(\boldsymbol{x} \rightarrow \tilde{\boldsymbol{x}} \mid \boldsymbol{h}_{S D}, \boldsymbol{H}_{S R}, \boldsymbol{h}_{R D}\right)\right],
\end{aligned}
$$

where $\boldsymbol{H}_{S R}=\left[\boldsymbol{h}_{S R_{1}}, \boldsymbol{h}_{S R_{2}}, \cdots, \boldsymbol{h}_{S R_{M}}\right]^{\mathrm{T}}$ is the channel fading matrix from sources to relays, the channel fading coefficient vector from the relays to the destination is denoted by $\boldsymbol{h}_{R D}=\left[h_{R_{1} D}, h_{R_{2} D}, \cdots, h_{R_{M} D}\right]^{\mathrm{T}}$. Given $\boldsymbol{h}_{S D}, \boldsymbol{H}_{S R}$ and $\boldsymbol{h}_{R D}$, $\operatorname{Pr}\left(\boldsymbol{x} \rightarrow \tilde{\boldsymbol{x}} \mid \boldsymbol{h}_{S D}, \boldsymbol{H}_{S R}, \boldsymbol{h}_{R D}\right)$ denotes the pair-wise error probability (PEP) at $D$ that $\tilde{\boldsymbol{x}}$ is received when $\boldsymbol{x}$ is transmitted, and the average PEP is given by

$$
\begin{aligned}
& E_{\boldsymbol{h}_{S D}, \boldsymbol{H}_{S R}, \boldsymbol{h}_{R D}}\left[\operatorname{Pr}\left(\boldsymbol{x} \rightarrow \tilde{\boldsymbol{x}} \mid \boldsymbol{h}_{S D}, \boldsymbol{H}_{S R}, \boldsymbol{h}_{R D}\right)\right]= \\
& \quad \sum_{\forall \mathcal{M},|\mathcal{M}|<K} E_{\boldsymbol{h}_{S D}}\left[\operatorname{Pr}\left(\boldsymbol{x} \rightarrow \tilde{\boldsymbol{x}} \mid \boldsymbol{h}_{S D}\right)\right] E_{\boldsymbol{H}_{S R}}\left[\operatorname{Pr}\left(\mathcal{M} \mid \boldsymbol{H}_{S R}\right)\right]+ \\
& \quad \sum_{\forall \mathcal{M},|\mathcal{M}| \geq K} E_{\boldsymbol{h}_{S D}, h_{R_{w} D}}\left[\operatorname{Pr}\left(\boldsymbol{x} \rightarrow \tilde{\boldsymbol{x}} \mid \boldsymbol{h}_{S D}, h_{R_{w} D}, \mathcal{M}\right)\right] \\
& E_{\boldsymbol{H}_{S R}}\left[\operatorname{Pr}\left(\mathcal{M} \mid \boldsymbol{H}_{S R}\right)\right],
\end{aligned}
$$

where $\operatorname{Pr}\left(\boldsymbol{x} \rightarrow \tilde{\boldsymbol{x}} \mid \boldsymbol{h}_{S D}\right)$ denotes the PEP at $D$ when no relay transmits signal to the destination in the relaying phase. $\operatorname{Pr}\left(\boldsymbol{x} \rightarrow \tilde{\boldsymbol{x}} \mid \boldsymbol{h}_{S D}, h_{R_{w} D}, \mathcal{M}\right)$ represents the PEP at $D$ when the $K$-th best relay $R_{w}$ is selected in the set $\mathcal{M}$. $\operatorname{Pr}\left(\mathcal{M} \mid \boldsymbol{H}_{S R}\right)$ represents the probability that the set of relays can successfully decode $\boldsymbol{x}$ is $\mathcal{M}$.

The probability $\operatorname{Pr}\left(\mathcal{M} \mid \boldsymbol{H}_{S R}\right)$ is given by

$$
\begin{aligned}
& \operatorname{Pr}\left(\mathcal{M} \mid \boldsymbol{H}_{S R}\right)=\prod_{m \in \mathcal{M}}\left(1-P_{\mathrm{e}}^{S R_{m}}\right) \prod_{m \notin \mathcal{M}} P_{\mathrm{e}}^{S R_{m}} \\
& \quad \leq\left\{\begin{array}{cc}
1, & \mathcal{M}=\mathbb{S}, \\
\prod_{m \notin \mathcal{M}} P_{\mathrm{e}}^{S R_{m}}, & \mathcal{M} \neq \mathbb{S},
\end{array}\right.
\end{aligned}
$$

where $P_{\mathrm{e}}^{S R_{m}}$ represents the decoding error probability at $R_{m}$, and it can be upper bounded by [20]

$$
\begin{aligned}
& P_{\mathrm{e}}^{S R_{m}} \leq \sum_{\forall \hat{\boldsymbol{x}}_{R_{m}} \in \mathcal{A}_{x}, \hat{\boldsymbol{x}}_{R_{m}} \neq \boldsymbol{x}} E_{\boldsymbol{h}_{S R_{m}}}\left[\operatorname{Pr}\left(\boldsymbol{x} \rightarrow \hat{\boldsymbol{x}}_{R_{m}} \mid \boldsymbol{h}_{S R_{m}}\right)\right]= \\
& \sum_{\substack{\forall \hat{x}_{R_{m} \in \mathcal{A}_{x}} \\
\hat{\boldsymbol{x}}_{R_{m}} \neq \boldsymbol{x}}} E_{\boldsymbol{h}_{S R_{m}}}\left[Q\left(\sqrt{\frac{\alpha \beta_{S}^{2} \bar{\gamma}\left\|\boldsymbol{h}_{S R_{m}} \boldsymbol{\theta}_{S}\left(\boldsymbol{x}-\hat{\boldsymbol{x}}_{R_{m}}\right)\right\|^{2}}{2}}\right)\right] \stackrel{\text { (a) }}{=} \\
& \sum_{\substack{\forall \hat{x}_{R_{m}} \in \mathcal{A}_{x} \\
\hat{x}_{R_{m}} \neq \boldsymbol{x}}} \frac{1}{\pi} \int_{0}^{\frac{\pi}{2}} \frac{1}{1+\frac{\alpha \beta_{S}^{2} \sigma_{S R}^{2} \bar{\gamma}}{4 \sin ^{2} \phi} \sum_{n=1}^{N}\left\|\theta_{S_{n}}\left(x_{n}-\hat{x}_{R_{m}, n}\right)\right\|^{2}} \mathrm{~d} \phi \\
& \stackrel{\bar{\gamma} \gg 1}{\approx} \frac{t_{R}}{\alpha \beta_{S}^{2} \sigma_{S R}^{2}} \bar{\gamma}^{-1},
\end{aligned}
$$

where $\operatorname{Pr}\left(\boldsymbol{x} \rightarrow \hat{\boldsymbol{x}}_{R_{m}} \mid \boldsymbol{h}_{S R_{m}}\right)$ represents the PEP at $R_{m}$, the average transmit signal to noise ratio (SNR) is denoted by $\bar{\gamma}=P / N_{0}$, and the Eq. (a) is from the following second facts [21]:

$$
\left\{\begin{array}{l}
Q(\sqrt{x})=\frac{1}{\pi} \int_{0}^{\frac{\pi}{2}} \exp \left(\frac{x}{2 \sin ^{2} \phi}\right) \mathrm{d} \phi, \\
E_{z}\left[\exp \left(-\boldsymbol{z}^{\mathrm{H}} \boldsymbol{A} \boldsymbol{z}\right)\right]=\operatorname{det}^{-1}(\boldsymbol{I}+\boldsymbol{\Xi} \boldsymbol{A}) .
\end{array}\right.
$$

where $\boldsymbol{z}$ is a column vector with $\boldsymbol{z} \sim \mathcal{C N}(0, \boldsymbol{\Xi})$ and $\boldsymbol{A}$ is a hermitian matrix.

Let $U_{1}, U_{2}, \cdots, U_{|\mathcal{M}|}$ be $|\mathcal{M}|$ i.i.d. exponential RVs 
with the same expectation $\sigma_{R D}^{2}$. Sort $|\mathcal{M}| \mathrm{RV}$ s in a non-increasing order $U_{(1)} \geq U_{(2)} \geq \cdots \geq U_{(M)}$; then $U_{(K)}$ is the $K$-th largest among all the RVs. The probability density function of $U_{(K)}$ is [22]

$$
f_{U_{K}}(u)=\frac{\sigma_{R D}^{-2} M ! \exp \left(-K \sigma_{R D}^{-2} u\right)\left[1-\exp \left(-\sigma_{R D}^{-2} u\right)\right]}{(M-K) !(K-1) !} .
$$

According to [18] and [19], the PEP at $D$ when the $K$-th best relay $R_{w}$ is selected in the set $\mathcal{M}$ is given by (12):

$$
\begin{aligned}
& E_{h_{S D}, h_{R_{w} D}}\left[\operatorname{Pr}\left(\boldsymbol{x} \rightarrow \tilde{\boldsymbol{x}} \mid \boldsymbol{h}_{S D}, h_{R_{w} D}, \mathcal{M}\right)\right]= \\
& \quad E_{h_{S D}, h_{R_{w} D}}\left[Q\left(\sqrt{\frac{\bar{\gamma}}{2}\left[\alpha \beta_{S}^{2}\left\|\boldsymbol{h}_{S D} \boldsymbol{\theta}_{S}(\boldsymbol{x}-\tilde{\boldsymbol{x}})\right\|^{2}+(1-\alpha) \beta_{R}^{2}\left\|h_{R_{w} D} \boldsymbol{\theta}_{R}(\boldsymbol{x}-\tilde{\boldsymbol{x}})\right\|^{2}\right]}\right)\right] \underset{\bar{\gamma} \gg 1}{\approx} f(|\mathcal{M}|) \cdot \bar{\gamma}^{-(|\mathcal{M}|-K+2)} .
\end{aligned}
$$

Similar to (11), the average PEP at $D$ when no relay forwards signal at the second phase, is given by

$$
E_{\boldsymbol{h}_{S D}}\left[\operatorname{Pr}\left(\boldsymbol{x} \rightarrow \tilde{\boldsymbol{x}} \mid \boldsymbol{h}_{S D}\right)\right] \stackrel{\bar{\gamma} \gg 1}{\approx} \frac{\bar{\gamma}^{-1}}{t_{1} \alpha \beta_{S}^{2} \sigma_{S D}^{2}} .
$$

Substituting (9)-(13) into (8) and we conclude the theorem.

Obviously, the diversity order of the CFNC scheme with $\mathrm{KBS}$ is $(M-K+2)$. Unlike the diversity order analysis only in [3-4], now the coding gain of the KBS with CFNC scheme in (7) can be utilized as the criterion in deriving the optimal power allocation factor $\alpha$, which will be elaborated in the next section.

\section{Optimized power allocation analysis}

In this section, the optimal power allocation for the CFNC scheme with KBS is derived in order to minimize the SEP in (6). However, due to the high computation complexity, the coding gain is simplified at first.

Let

$$
\begin{aligned}
& d_{\min }^{D_{1}}=\min _{\forall x_{n}, \tilde{x}_{n} \in C_{n}, x_{n} \neq \tilde{x}_{n}}\left\|\theta_{S_{n}}\left(x_{n}-\tilde{x}_{n}\right)\right\|, \text { and } \\
& d_{\min }^{D_{2}}=\min _{\forall \boldsymbol{x}, \tilde{\boldsymbol{x}} \in \mathcal{A}_{x}, \boldsymbol{x} \neq \tilde{\boldsymbol{x}}}\left\|\boldsymbol{\theta}_{R}(\boldsymbol{x}-\tilde{\boldsymbol{x}})\right\| .
\end{aligned}
$$

We have

$$
\begin{aligned}
& \sum_{n=1}^{N}\left\|\theta_{S_{n}}\left(x_{n}-\tilde{x}_{n}\right)\right\|^{2} \geq\left(d_{\min }^{D_{1}}\right)^{2}, \\
& \left\|\boldsymbol{\theta}_{R}(\boldsymbol{x}-\tilde{\boldsymbol{x}})\right\|^{2} \geq\left(d_{\text {min }}^{D_{2}}\right)^{2}, \text { and } \\
& \sum_{n=1}^{N}\left\|\theta_{S_{n}}\left(x_{n}-\hat{x}_{R_{m}, n}\right)\right\|^{2} \geq\left(d_{\min }^{D_{1}}\right)^{2} .
\end{aligned}
$$

Then the coding gain in (7) can be relaxed as

$$
G_{c}^{s} \stackrel{\bar{\gamma} \gg 1}{\approx}\left(\frac{\sigma_{S R}^{2}}{\sigma_{S D}^{2}} \kappa_{2}(\alpha)\right)^{-\frac{1}{M-K+2}},
$$

where

$$
\begin{aligned}
& \kappa_{2}(\alpha)=\sum_{i=K}^{M} a_{i}\left(\frac{b}{1-\alpha}\right)^{i-K+1}\left(\frac{c}{\alpha}\right)^{M-i+1}+ \\
& \left(\begin{array}{l}
M \\
K-1
\end{array}\right)\left(\frac{c}{\alpha}\right)^{M-K+2}, \\
& a_{i}=\frac{2 i !\left(\begin{array}{c}
M \\
i
\end{array}\right) \Gamma(i-K+1)(2(i-K)+3) ! !}{(i-K) !(K-1) !(2(i-L+2)) ! !}, \\
& b=\frac{4}{\beta_{R}^{2} \sigma_{R D}^{2}\left(d_{\min }^{D_{2}}\right)^{2}}, \text { and } c=\frac{\left|\mathcal{A}_{x}\right|-1}{\beta_{S}^{2} \sigma_{S R}^{2}\left(d_{\min }^{D_{1}}\right)^{2}} .
\end{aligned}
$$

Theorem 2 If the function $\kappa_{2}(\alpha)$ is strictly convex on the open set $(0,1)$, then $\kappa_{2}(\alpha)$ on $(0,1)$ has one global minimum value.

\section{Proof:}

Firstly, the second derivatives of $\kappa_{2}(\alpha)$ is given by

$$
\begin{aligned}
& \frac{\partial^{2} \kappa_{2}(\alpha)}{\partial \alpha^{2}}= \\
& \sum_{i=K}^{M} a_{i} b^{i-K+1} c^{M-i+1} g_{i}(\alpha) \alpha^{-(M-i+3)}(1-\alpha)^{-(i-K+1)}+ \\
& \left(\begin{array}{l}
M \\
K-1
\end{array}\right)(M-K+2)(M-K+3) c^{M-K+2} \alpha^{-(M-K+4)},
\end{aligned}
$$

where

$$
\begin{aligned}
& g_{i}(\alpha)=(M-i+1)(M-i+2)(1-\alpha)^{2}+ \\
& \quad(i-K+1)(i-K+2) \alpha^{2}- \\
& 2(M-i+1)(i-K+1) \alpha(1-\alpha) .
\end{aligned}
$$

We will have the following second derivatives of $g_{i}(\alpha)$ as follows:

$\frac{\partial^{2} g_{i}(\alpha)}{\partial \alpha^{2}}=2(M-K+2)(M-K+3)>0$. 
From (17), it can be seen that the function $g_{i}(\alpha)$ is strictly convex, and then there must exist a minimum value within $(0,1)$ such that $g_{i}^{\min }(\alpha) \leq g_{i}(\alpha)$, and the minimum value is given by

$$
g_{i}^{\min }(\alpha)=\frac{(M-i+1)(i-K+1)}{M-K+2}>0 .
$$

Substituting (18) into (16) yields $\partial^{2} \kappa_{2}(\alpha) / \partial \alpha^{2}>0$. This completes Theorem 2 .

In order to minimize the SEP, the optimal allocation factor $\alpha$ can be derived from the following minimization problem:

$$
\begin{aligned}
P_{1}: & \min \kappa_{2}(\alpha), \\
& \text { subject to } 0<\alpha<1 .
\end{aligned}
$$

Because of the convexity in function $\kappa_{2}(\alpha)$ within the interval $(0,1)$, the optimal power allocation factor $\alpha_{\text {opt }}$ can be determined through numerical analysis, and some results are listed in Table 1.

Table 1 Optimal power allocation factor $\alpha_{\text {opt }}$ in $(3,5,1)$

\begin{tabular}{cccccccc}
\hline$\sigma_{S R}^{2}$ & \multicolumn{3}{c}{ BPSK } & & \multicolumn{3}{c}{ QPSK } \\
\cline { 2 - 3 } \cline { 7 - 8 }$\sigma_{R D}^{2}$ & $K=1$ & $K=3$ & $K=5$ & & $K=1$ & $K=3$ & $K=5$ \\
\hline 0.1 & 0.6797 & 0.7372 & 0.8261 & 0.7656 & 0.8060 & 0.8679 \\
1.0 & 0.3745 & 0.4663 & 0.6582 & 0.4622 & 0.5504 & 0.7124 \\
10 & 0.2121 & 0.2959 & 0.5358 & 0.2445 & 0.3305 & 0.5623 \\
\hline
\end{tabular}

Note: BPSK means binary phase shift keying, and QPSK means quadrature phase shift keying.

As shown in Table 1, more power should be allocated to the relaying phase with the improved source-relay link. With the increase in parameter $K$, more power should be allocated to MA phase as well. This can be intuitively explained by the fact that, more power should be allocated in the MA phase to improve the cardinality of set $|\mathcal{M}|$, and increase the probability that the $K$-th best relay is selected successfully.

\section{Simulations}

In this section, simulations are performed to verify the analysis in Section 3 and Section 4. Unless otherwise specified, $\alpha=0.5, \sigma_{S R}^{2}=\sigma_{S D}^{2}=\sigma_{R D}^{2}=1$, and BPSK modulation are assumed. In all simulations we consider a $(3,5,1)$ cooperative communication network. From the figures, it can be seen that the upper bound analysis fits the simulation results very well within high $P / N_{0}$ region.

The impact of parameter $K$ on system performance with optimal power allocation is shown in Fig. 1. As

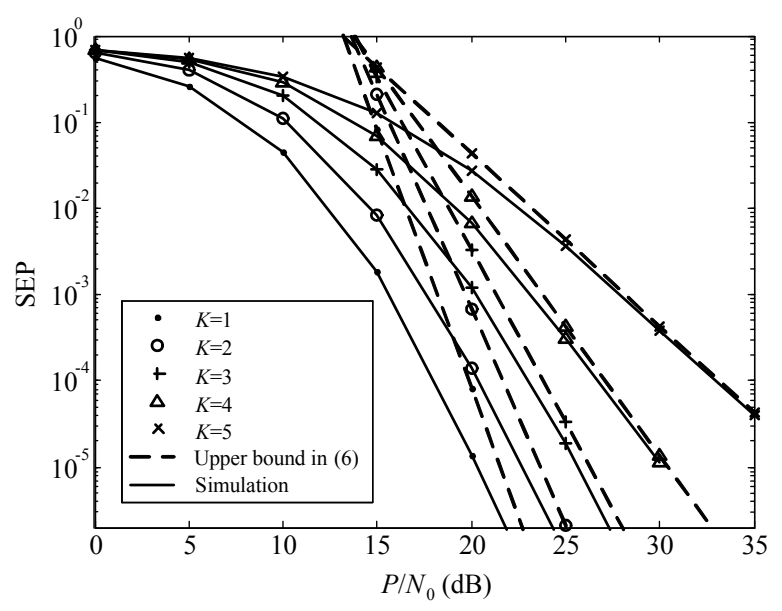

Fig. 1 Performance of $(3,5,1)$ network with optimal power allocation for different $K$

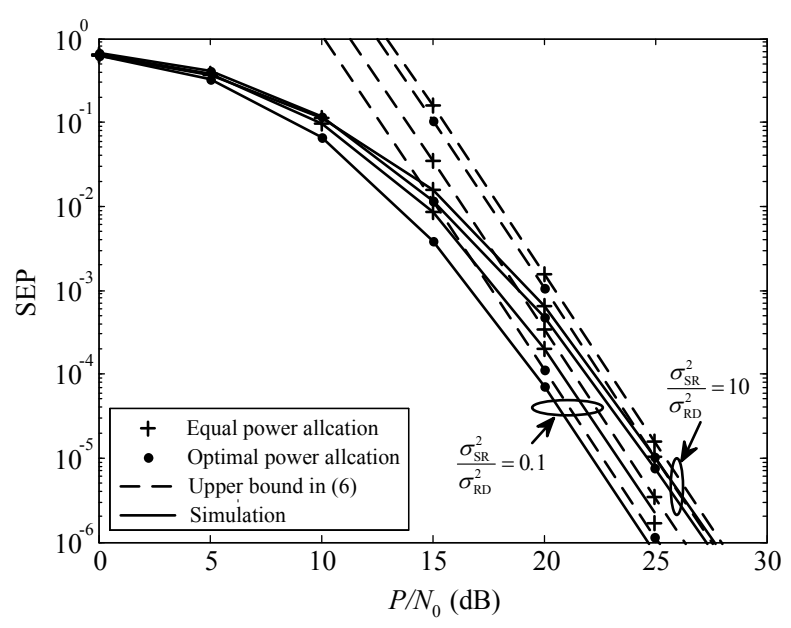

Fig. 2 Performance of $(3,5,1)$ network with equal and optimal power allocation $(K=3)$

one can see, the CFNC with KBS can achieve full diversity only when $K=1$, while the diversity order decreases with increasing parameter $K$. The performance of CFNC with different power allocation method is presented in Fig. 2. The SEP performance suggests that the optimal power allocation brings performance enhancement than equal allocation, given the total transmit power constraint at the sources and relays.

\section{Conclusions}

In this paper, for the CFNC scheme with KBR the union bound on SEP performance is derived and the optimal power allocation method is investigated. The union bound is used to optimally allocate the power among source nodes and relay node in MA and relaying phases. The numerical and simulation results show that the CFNC scheme with KBR can be significantly improved by using the optimal power allocation; the diversity or- 
der of the CFNC scheme with $\mathrm{KBR}$ is $(M-K+1)$; and the CFNC scheme with KBR could achieve full diversity only when $K=1$.

\section{Acknowledgements}

This work was supported by the Major State Basic Research Development Program of China (973 Program No. 2012CB316100), and the National Natural Science Foundation of China (Nos. 61032002/61271246), and the 111 Project (No. 111-2-14).

\section{References}

[1] J.N. Laneman, D.N.C. Tse, G.W. Wornell, Cooperative diversity in wireless networks: Efficient protocols and outage behavior, IEEE Transaction Information Theory, 2004, 50(12): 3062- 3080.

[2] T. Wang, G.B. Giannakis, R. Wang, Smart regenerative relays for link-adaptive cooperative communications. IEEE Transaction on Communications, 2008, 56(11): 1950-1960.

[3] T. Wang, G. Giannakis, Complex field network coding for multiuser cooperative communications, IEEE Selected Areas in Communication, 2008, 26(3): 561-571.

[4] T. Wang, G.B. Giannakis, High-throughput cooperative communications with complex field network coding. In: Proceedings of Conference on Information Sciences and Systems (CISS'07), The Johns Hopkins University, 2007: 253-258.

[5] Y. Wu, P. Chou, S. Kung, Information exchange in wireless networks with network coding and physical-layer broadcast. In: Proceedings of 2005 Conference on Information Sciences and Systems, The Johns Hopkins University, 2005: 16-18.

[6] Y. Chen, S. Kishore, J. Li, Wireless diversity through network coding, In: Proceedings of 2006 IEEE Wireless Communications and Networking Conference (WCNC'06), Las Vegas, NV USA, 2006: 1681-1686.

[7] L. Xiao, T. Fuja, J. Kliewer, et al., A network coding approach to cooperative diversity, IEEE Transaction Information Theory, 2007, 53(10): 3714-3722.

[8] X. Bao, J. Li, Adaptive network coded cooperation (ANCC) for wireless relay networks: matching code-ongraph with network-on-graph, IEEE Transactions on Wireless Communications, 2008, 7(2): 574-583.

[9] M. Peng, H. Liu, W. Wang, et al., Cooperative network coding with MIMO transmission in wireless decode-andforward relay networks, IEEE Transactions on Vehicular Technology, 2010, 59(7): 3577-3588.
[10] S. Zhang, S. Liew, P. Lam, Physical layer network coding, In: Proceeding of the Twelfth Annual International Conference on Mobile Computing and Network (ACM MobiCom'06), Los Angeles, CA, USA, 2006: 63-68.

[11] S. Katti, S. Gollakota, D. Katabi, Embracing wireless interference: analog network coding, In: Proceeding of the Flagship Annual Meeting of the Special Interest Group on Data Communication (ACM SIGCOMM '07), Kyoto, Japan, 2007: 397-408.

[12] L. Venturino, X. Wang, M. Lops, Multiuser detection for cooperative networks and performance analysis, IEEE Transactions on Signal Processing, 2006, 54(9): 3315-3329.

[13] L. Venturino, X. Wang, M. Lops, Multiuser detection for cooperative networks and performance analysis, In: Proceeding of the IEEE Signal Processing Workshop on Signal Processing Advances in Wireless Communications (SPAWC'05), New York, USA, 2005: 940-944.

[14] Z. Han, X. Zhang, H.V. Win, Cooperative transmission protocols with high spectral efficiency and high diversity order using multiuser detection and network coding. In: Proceeding of IEEE International Conference on Communications (ICC'07), Grasse, 2007: 4232-4236.

[15] M. Ju, I.M. Kim, Relay selection with ANC and TDBC protocols in bidirectional relay networks, IEEE Transaction on Communications, 2010, 58(12): 3500-3511.

[16] A. Bletsas, A. Khisti, D.P. Reed, et al., A simple cooperative diversity method based on network path selection, IEEE Journal on Selected Areas Communications, 2006, 24(3): 659-67.

[17] A. Bletsas, H. Shin, M.Z. Win, Cooperative communications with outage-optimal opportunistic relaying, IEEE Transactions on Wireless Communications, 2007, 6(9): 3450-3460.

[18] S.S. Ikki, M.H. Ahmed, On the performance of cooperative-diversity networks with the $N$-th best-relay selection scheme, IEEE Transactions on Communications, 2010, 58(11): 3062-3069.

[19] J. Tian, Q. Zhang, S. H. Ting, LCR and AFD of Decodeand Forward Relay Networks with $N^{\text {th }}$ Best Relay Selection Schemes in Rayleigh Fading Channels, IEEE Wireless Communications Letters, 2012, 1(4): 380-383.

[20] A. Cano, T. Wang, A Ribeiro, et al., Link-adaptive distributed coding for multisource cooperation, EURASIP Journal on Advances in Signal Processing, 2008, 38: 1-12.

[21] Y. Ding, J.K. Zhang, K.M. Wong, The amplify-andforward half-duplex cooperative system: pairwise error probability and precoder design, IEEE Transactions on Signal Processing, 2007, 55(2): 605-617.

[22] Q.F. Zhou, Y. Li, F.C.M. Lau, et al. Decode-and-forward two-way relaying with network coding and opportunistic relay selection, IEEE Transactions on Communications, 2010, 58(11): 3070-3076.

(Editor: Junsi LAN) 